\title{
Bildgebungsverfahren decken hohe Fehlerquote auf
}

\author{
Wachkoma-Patienten teilweise kontaktfähiger als bisher vermutet
}

In Deutschland leben etwa 5.000 Wachkoma-Patienten, in Österreich sind es zwischen 800 und 1.000. Die korrekte Diagnose des genauen Bewusstseinszustands ist jedoch eine klinische Herausforderung. Denn erfolgt sie ohne spezielle Bewusstseinsskalen, liegt die Rate der Fehldiagnosen bei etwa 40 Prozent. Aktuelle Studien mithilfe hochauflösender Elektroenzephalographie (EEG) und funktioneller Kernspintomographie (fMRT) zeigen zudem, dass Ärzte die Reaktionsfähigkeit häufig falsch einschätzen, so die Deutsche Gesellschaft für Klinische Neurophysiologie und funktionelle Bildgebung (DGKN). Jeder fünfte Wachkoma-Patient weist etwa in speziellen EEG-Verfahren Hinweise für bewusste Reaktionen auf, die Standardverfahren nicht erkennen. Die DGKN fordert daher, funktionelle Bildgebungsverfahren sowie spezielle elektrophysiologische Verfahren bei der Beurteilung von Wachkoma-Patienten häufiger einzusetzen.

Wachkoma-Patienten zeigen kein Bewusstsein für ihre Umgebung oder sich selbst, obwohl ihre Augen offen sind. Um die Reaktionsfähigkeit dieser Patienten zu ermitteln, sollten Ärzte Bewusstseinsskalen nutzen. Das international bewährte und standardisierte klinische Diagnoseinstrument, die sogenannte Coma Recovery Scale-Revised (CRS-R), kommt nach Schätzungen der DGKN aber bisher bei weniger als fünf Prozent der WachkomaPatienten zum Einsatz. Mit dem CRS-R untersuchen Ärzte systematisch akustische, visuelle, motorische und verbale Reaktionen auf Reize, sowie den Aktivierungsgrad des Nervensystems. „Neueste Erkenntnisse zeigen jedoch, dass wir unser Verständnis vom Wachkoma grundlegend verändern müssen", erklärt Privatdozent Dr. med. Andreas Bender, Leiter des Therapiezentrums Burgau in Bayern und Spezialist für Hirnschäden.

Aktuelle Studien deuten darauf hin, dass elektrophysiologische und bildgebende Verfahren wie EEG und fMRT zusätzliche Reaktionen bei WachkomaPatienten messen, die Verhaltensbeobachtungen von außen nicht erkennen lassen. So weisen etwa 17 Prozent der
Wachkoma-Patienten typische Aktivierungsmuster vergleichbar mit jenen gesunder Probanden im fMRT auf. „Teilweise war sogar eine einfache Kommunikation mithilfe des fMRT-Signals möglich“, so der Experte der DGKN. Dabei konnte ein Patient während der fMRT-Untersuchung richtige Ja- oder Nein-Antworten auf autobiographische Fragen geben (zum Beispiel „Ist der Name Ihres Vaters Alexander?"). Bildgebende Verfahren hätten daher in den vergangenen Jahren einen Paradigmenwechsel bei der Beurteilung von Wachkoma-Patienten hervorgerufen.

Da die fMRT allerdings sehr aufwändig, teuer und störanfällig ist, bietet sich das EEG als alltagstauglichere Untersuchungsmethode zur Prüfung der Hirnfunktion der Betroffenen an. „Auch mit dieser Methode konnten eindrucksvolle Erkenntnisse gewonnen werden: Bei verbalen Aufforderungen und Tonsignalen werden bestimmte Hirnregionen aktiv, und es kommt zu einer Veränderung des EEG-Frequenzspektrums", so Bender. In einer aktuellen Studie reagierten drei von 16 Wachkoma-Patienten auf verbale Aufforderungen, die mit der CRS-R nicht erfasst wurden.

„Unterhalb der Schwelle der klinischen Beobachtbarkeit mit Komaskalen gibt es somit bei etwa jedem fünften bis sechsten Patienten eindeutige Hinweise für bewusste Interaktionen mit der Umwelt“, fasst Bender die Ergebnisse zusammen. Die Rate der Fehldiagnosen bei Wachkoma-Patienten liege daher sicher höher als bisher vermutet, schätzen Experten der DGKN. Der routinemäßige Einsatz der funktionellen Bildgebung bei Koma-Patienten sei derzeit zwar verfrüht. Doch ließen sich aus diesen Erkenntnissen der Bewusstseinsforschung künftig neue Therapien ableiten, wie beispielsweise die Entwicklung von Brain-Computer-Interfaces für eine Kommunikation mit KomaPatienten.

Quelle: Presseaussendung der Deutschen Gesellschaft für klinische Neurophysiologie

\section{LITERATUR}

Monti MM, Vanhaudenhuyse A, Coleman MR, Boly M, Pickard JD, Tshibanda L, Owen AM, Laureys S. Willful modulation of brain activity in disorders of consciousness. N Engl J Med. 2010 Feb 18;362(7):579-89.

Cruse D, Chennu S, Chatelle C, Bekinschtein TA, Fernández-Espejo D, Pickard JD, Laureys S, Owen AM. Bedside detection of awareness in the vegetative state: a cohort study. Lancet. 2011 Nov 9.

Owen AM, Coleman MR, Boly M, Davis MH,

Laureys S, Pickard JD. Detecting awareness in the vegetative state. Science. 2006 Sep 8;313(5792):1402.

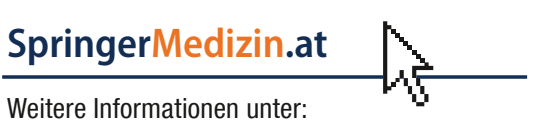
www.SpringerMedizin.at
Buchempfehlung

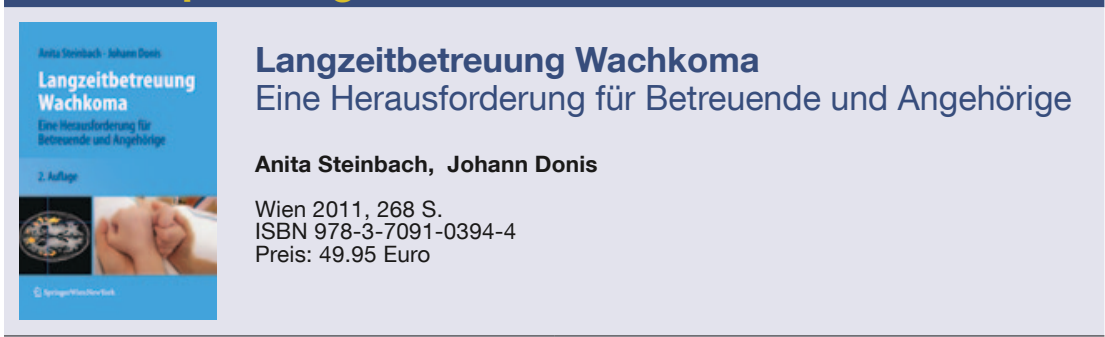

Das Thema Wachkoma hat in den vergangenen Jahren zunehmend Aufmerksamkeit auf sich gezogen. Kein anderes Krankheitsbild fordert das interdisziplinäre Team mehr als die Betreuung eines Menschen in diesem auf unbestimmte Zeit reduzierten Bewusstseinszustand. Neueste Forschungsergebnisse führen zu einem Paradigmenwechsel im Umgang mit Menschen im Wachkoma. Es gilt, strukturelle Voraussetzungen zu schaffen, theoriegeleitet zu handeln und damit sicherzustellen, dass subjektive Interpretationen und Meinungen auf eine objektivierbare Basis gestellt werden. 